\title{
Modeling and Control for a Near-space Morphing Glide Aircraft
}

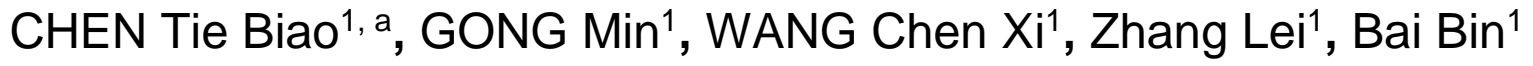 \\ ${ }^{1}$ China Acadamy of Launch Vehicle Technology, China \\ a18515490333@163.com
}

\begin{abstract}
Keywords: near-space glide aircraft; morphing aircraft; auto disturbance rejection controller Abstract. Aaccording to the morphing requirements, this article proposes a morphing glide aircraft with high environmental adaptability. Based on Kane's Method, six degree-of freedom dynamic model is built. The automatic disturbance rejection control technology is adopted to realize stability control of longitudinal model of MGA, that provides a reference for the control technology research of morphing aircraft.
\end{abstract}

\section{Introduction}

In May 2017, NASA officially reported the second flight test of " the adaptive flexible trailing edge" project, indicating that flexible morphing wing technology has entered the stage of engineering application. With the technical traction of "artificial intelligence" and the demand of intelligent warfare, aircraft needs not only a "smart brain" which can make strategic decisions in the future, but also a body which can adapt to changes of complex battlefield environment. The near-space glide aircraft is the typical representative of hypersonic aircraft, which has the advantages of long range, quick response, high precision and strong penetration ability. However, the dramatic change of flight environment and the geometric shape of the big wingspan bring the following technology difficulties:(1) Poor environment adaptability of entire flight profile. (2) Design difficulties for control systems and separation systems in large dynamic pressure. (3) Difficulties to meet the geometric constraints of shipborne launch and airborne launch. The application of morphing technology to the near space glide aircraft has the following advantages:

(1) Improve the environmental adaptability of the aircraft through morphing to achieve the optimal performance in large airspace and wide speed range;

(2) Reduce the static instability of the boost flight and the design difficulty of the control system through morphing;

(3) Reduce the span dimension through morphing, which is convenient for transportation and launching;

(4) Realize the redundant attitude control through morphing, which can improve the fault-tolerant ability of the control system;

(5) Expand mission mode and flight envelope through morphing, which can improve the penetration capability of aircraft;

(6) Realize intelligent combat through adaptive morphing and intelligent decision.

A great deal of fruitful work on morphing aircraft have been done by Scholars. NextGen Airlines studied the wing shape control and flight control of MFX [1]. Florida University designed a model tracking control algorithm to maintain optimal flight performance in the four flight stages of the morphing aircraft: cruising, maneuvering, steeply descending and tracking[2]. Cornell University studied trajectory optimization of morphing aircraft [3]. At present, the research on morphing aircraft is mainly aimed at low speed aircraft, and there are few reports about the related research of near-space glide aircraft with greater morphing demand.

This paper proposes a kind of variable-span morphing glide aircraft (MGA), which is easy to apply on the engineering. The Kane's method is adopted to establish MGA's six degree-of-freedoms (DOF) dynamic model. And then, for the longitudinal model, the Auto disturbance rejection controller (ADRC) is designed to keep stable before, during and after the morphing of MGA. 


\section{Kane's Method}

Kane's Method uses generalized speeds as the independent variables to describe the system's movement in replace of the generalized coordinates. For multi-rigid systems, Kane's Method chooses $\mathrm{f}$ ( $\mathrm{f}$ is number of system's DOF) independent generalized velocities as the independent variables to describe the system. So, any rigid body in the system can be uniquely expressed as a linear combination of generalized speeds:

$$
\left\{\begin{array}{l}
v_{c i}=\sum_{\gamma=1}^{f} v_{i \gamma}^{\prime} \pi \delta \gamma+v_{i 0}^{\prime} \\
\omega_{i}=\sum_{\gamma=1}^{f} \omega_{i \gamma}^{\prime} \pi \delta \gamma+\omega_{i 0}^{\prime}
\end{array}(\gamma=1,2 \mathrm{~L} f) .\right.
$$

$v_{i \gamma}^{\prime} \omega_{i \gamma}^{\prime}$ are partial velocity and partial angle velocity of rigid body $\gamma$.

For a system composed of $\mathrm{N}$ rigid bodies with $\mathrm{f}$ degrees of freedom, the Kane equation is expressed in the inertial reference coordinate system as follows[4]:

$$
\left\{\begin{array}{l}
K_{\gamma}+K_{\gamma}^{*}=0 \\
K_{\gamma}=\sum_{i=1}^{N}\left(F_{i} \cdot v_{i \gamma}^{\prime}+M_{i} \cdot \omega_{i \gamma}^{\prime}\right) \quad(\gamma=1,2 \mathrm{~L} f) . \\
K_{\gamma}^{*}=\sum_{i=1}^{N}\left(F_{i}^{*} \cdot v_{i \gamma}^{\prime}+M_{i}^{*} \cdot \omega_{i \gamma}^{\prime}\right)
\end{array}\right.
$$

Where $K_{\gamma}$ and $K_{\gamma}^{*}$ are generalized applied force and generalized inertial force $. F_{i}, M_{i}$ are the applied force and the applied moment relative to center of mass acting on rigid body i, $F_{i}^{*}, M_{i}^{*}$ are the inertial force and the inertial moment relative to center of mass acting on rigid body i. $F_{i}^{*}, M_{i}^{*}$ can be obtained by the following formula:

$$
\left\{\begin{array}{l}
F_{i}^{*}=-m_{i} a_{c i} \\
M_{i}^{*}=-J_{i} \cdot \omega_{i}-\omega_{i} \times\left(J_{i} \cdot \omega_{i}\right)
\end{array}(i=1,2 \mathrm{~L} N) .\right.
$$

Where $J_{i}$ is the inertia tensor of the body $\mathrm{i}, a_{c i}$ is the acceleration of center of mass of rigid body $\mathrm{i}$.

\section{Dynamic Model}

Physical model simplification. The MGA is composed of a fuselage F (body1), inner part of left morphing wing WL1 (body2), outer part of left morphing wing WL2 (body3), inner part of right morphing wing WR1 (body4), and outer part of right morphing wing WR2 (body5). The mass of the body1 is $M_{F}$, and the center of mass is $O_{1}$. Four morphing wings can be simplified into a parallelogram plate with mass $M_{W}$, length $l$, sweep angle $\varsigma$, center of mass OL1 、OL2 、OR1、 OR2. The MGA with total mass $M_{T}$, center of mass $O_{T}$, can morph continuously during flight, the length that body 2 stretchs out from body 1 is $\Delta_{1}$, the length that body3 stretchs out from body 2 is $\Delta_{2}$, the length that body 4 stretchs out from body 3 is $\Delta_{3}$, the length that body5 stretch out from body 4 is 
$\Delta_{4}$, the total stretch length on the left is $\Delta_{L}=\Delta_{1}+\Delta_{3}$, the total stretch length on the right is $\Delta_{R}=\Delta_{2}+\Delta_{4}$.

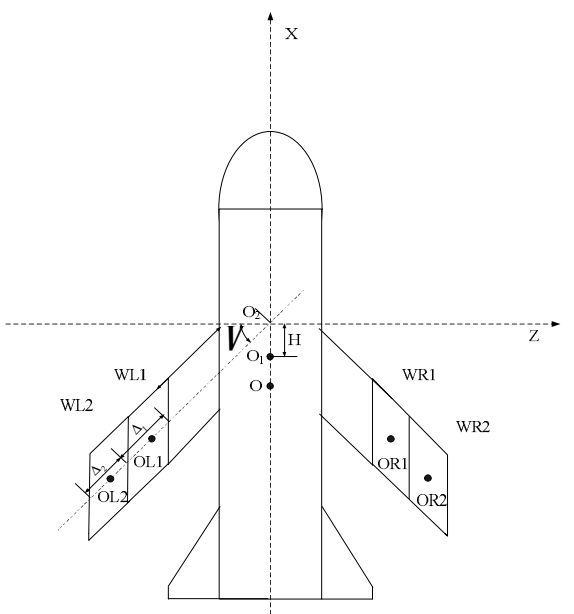

Fig. 1 Morphing glide aircraft

Definition of the coordinate Frames. The frames used in this paper are show in Fig. 2, because the center of mass of MGA $O_{T}$ will change during the morphing progress, $O_{1}$ is selected as the origin of the body-fixed frame $O_{1}-X_{b} Y_{b} Z_{b}$ and airstream frame $O_{1}-X_{v} Y_{v} Z_{v}$. The $O_{1} X_{v}$ axis direction of the airstream frame $O_{1}-X_{v} Y_{v} Z_{v}$ is corresponding to the velocity of the aircraft. $O-X_{o} Y_{o} Z_{o}$ is the inertia ground frame. $O_{L 1}-X_{L 1} Y_{L 1} Z_{L 1}$ is fixed to body2 with OL1 as origin; $O_{L 2}-X_{L 2} Y_{L 2} Z_{L 2}$ is fixed to body3 with OL2 as origin; $O_{R 1}-X_{R 1} Y_{R 1} Z_{R 1}$ is fixed to body4 with OR1 as origin; $O_{R 2}-X_{R 2} Y_{R 2} Z_{R 2}$ is fixed to body 5 with OR 2 as origin. The relative orientation of frame $i$ and frame $j$ can be expressed by a matrix Aij.

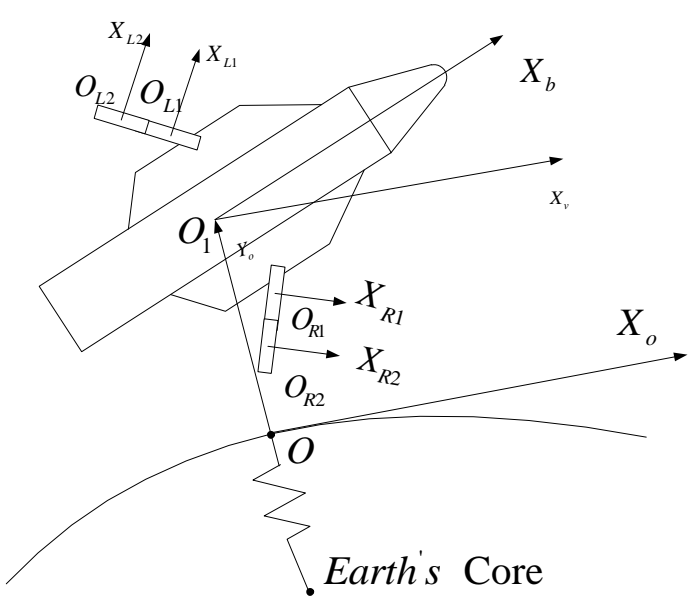

Fig. 2 Coordinate system diagram

Deflecting velocity and angular velocity. In this paper, we assume that the wing's morphing motion relative to fuselage can be controlled effectively by the morphing control system. The position, velocity and acceleration of the wing's span can be controlled to the desired values. Then a given morphing process can be expressed by a set of constraint equations as follows: 


$$
\Delta_{i}=f\left(\Delta_{i}^{*}, t\right), i=(1,2,3,4)
$$

where $\Delta_{i}$ are the state vector, denote the state of the wing's morphing motion, and $\Delta_{i}^{*}$ are the desired values of these states. Equation (4) can express the DOF related to the morphing motion, and the DOF of the MGA is reduced to six ( The coordinate of $O_{1}$ in $O-X_{o} Y_{o} Z_{o}$, which are x, y, z. The orientation angles of $O_{1}-X_{b} Y_{b} Z_{b}$ relative to $O-X_{o} Y_{o} Z_{o}$, which are pitch angle $\theta$, yaw angle $\psi$ and roll angle $\phi)$. We choose the six variables $u, v, w, p, q, r$ as the generalized speeds $\pi_{\gamma}(\gamma=1,2, \mathrm{~L} 6)$. Then, the partial velocity and partial angular velocity of the center of mass can be obtained according to Equation (1).

Applied Force and Generalized Inertial Force. According to the assumption as Equation (4), the interactional forces between morphing wings and fuselage, and the interactional forces between the inner part and the outer parts of the morphing wings can be treated as constraint forces, which will not be included in the Kane's equations obviously.

The applied forces include gravity and aerodynamic power. The aerodynamic force and moment acting on the aircraft is completely simplified to the fuselage. The applied moment of the four morphing wings is zero and the applied force is only gravity.

$$
\begin{aligned}
& F_{1}=\left[\begin{array}{l}
-M_{F} g \sin \theta \cos \psi-D \cos \beta \cos \alpha+L \sin \alpha-Y \sin \beta \cos \alpha \\
-M_{F} g(\sin \theta \sin \psi \sin \phi+\cos \theta \cos \phi)+D \cos \beta \sin \alpha+L \cos \alpha+Y \sin \beta \sin \alpha \\
-M_{F} g(\sin \theta \sin \psi \cos \phi-\cos \theta \sin \phi)-D \sin \beta+Y \cos \beta
\end{array}\right] \\
& F_{L 1}=F_{L 2}=F_{R 1}=F_{R 2}=\left[\begin{array}{c}
-M_{W} g \sin \theta \cos \psi \\
-M_{W} g(\sin \theta \sin \psi \sin \phi+\cos \theta \cos \phi) \\
-M_{W} g(\sin \theta \sin \psi \cos \phi-\cos \theta \sin \phi)
\end{array}\right] \\
& T_{1}=\left[\begin{array}{lll}
M_{x} & M_{y} & M_{z}
\end{array}\right]^{T} \\
& T_{L 1}=T_{L 2}=T_{R 1}=T_{R 2}=\left[\begin{array}{lll}
0 & 0 & 0
\end{array}\right]^{T}
\end{aligned}
$$

Where $D$ is the resistance, $L$ is the lift, $Y$ is the lateral force, $M_{x}$ is the roll moment, $M_{y}$ is the yaw moment, and $M_{z}$ is the pitch moment.

Generalized inertial force and generalized inertial moment can be calculated according to Equation (3).

Based on Equation (2), dynamic equations of MGA are achieved, as the equations (6) - (11), which are built in frame $O_{1}-X_{b} Y_{b} Z_{b}$. Equations (6) - (8) express the balance of forces, equations (9) - (11) express the balance of moment. $\alpha$ is the angle of attack, $\beta$ is the sideslip angle.

$$
\begin{aligned}
& 0=-M_{T}(g \sin \theta \cos \psi+\imath \&+q w-r v)-D \cos \beta \cos \alpha+L \sin \alpha-Y \sin \beta \cos \alpha \\
& +4 M_{w} H\left(r^{2}+q^{2}\right) \\
& -M_{w}\left(-\sin \eta \alpha_{1}^{\alpha}-\cos \eta\left(\mathbb{k}_{1}+2 q \phi_{1}^{\alpha}\right)+q^{2} L_{1} \sin \eta+r^{2} L_{1} \sin \eta-r p L_{1} \cos \eta\right) \\
& -M_{w}\left(-\sin \eta \otimes_{L}^{\alpha}-\cos \eta\left(\&_{2}+2 q \otimes_{L}\right)+q^{2} L_{2} \sin \eta+r^{2} L_{2} \sin \eta-r p L_{2} \cos \eta\right) \\
& -M_{w}\left(-\sin \eta{ }_{3}+\cos \eta\left(\alpha_{3}+2 q \alpha_{3}\right)+q^{2} L_{3} \sin \eta+r^{2} L_{3} \sin \eta+r p L_{3} \cos \eta\right) \\
& -M_{w}\left(-\sin \eta \Phi_{R}+\cos \eta\left(\alpha_{4}+2 q \S_{R}\right)+q^{2} L_{4} \sin \eta+r^{2} L_{4} \sin \eta+r p L_{4} \cos \eta\right)
\end{aligned}
$$




$$
\begin{aligned}
& 0=-M_{T}(g \sin \theta \sin \psi \sin \phi+g \cos \theta \cos \phi+\alpha w+r u)+D \cos \beta \sin \alpha+L \cos \alpha \\
& +Y \sin \beta \sin \alpha-4 M_{W} H(\&+p q) \\
& -M_{W}\left(-\sin \eta\left(\&_{1}+r \S_{1}\right)+\cos \eta\left(\alpha_{1}+2 p \S_{1}\right)-p q L_{1} \sin \eta-q r L_{1} \cos \eta-r \S_{1} \sin \eta\right) \\
& -M_{W}\left(-\sin \eta\left(\&_{2}+r \otimes_{L}\right)+\cos \eta\left(\alpha_{2}+2 p \otimes_{L}\right)-p q L_{2} \sin \eta-q r L_{2} \cos \eta-r \otimes_{L} \sin \eta\right) . \\
& -M_{W}\left(-\sin \eta\left(\varepsilon_{3}+r \otimes_{3}\right)-\cos \eta\left(\alpha_{3}+2 p \S_{3}\right)-p q L_{3} \sin \eta+q r L_{3} \cos \eta-r \otimes_{3} \sin \eta\right) \\
& -M_{W}\left(-\sin \eta\left(\&_{4}+r \otimes_{R}\right)-\cos \eta\left(\alpha_{4}+2 p \bigotimes_{R}\right)-p q L_{4} \sin \eta+q r L_{4} \cos \eta-r \otimes_{R} \sin \eta\right) \\
& 0=-M_{T}(g \sin \theta \sin \psi \cos \phi-g \cos \theta \sin \phi+\imath \alpha+p v-q u)-D \sin \beta+Y \cos \beta \\
& -4 M_{W} H(r p-\phi \\
& -M_{W}\left(-\cos \eta \Phi_{1}+\sin \eta\left(\alpha_{1}+2 q \otimes_{1}\right)-r p L_{1} \sin \eta+\left(p^{2}+q^{2}\right) L_{1} \cos \eta\right) \\
& -M_{W}\left(-\cos \eta \&_{L}^{\alpha}+\sin \eta\left(\phi \mathbb{k}_{2}+2 q \boldsymbol{\otimes}_{L}\right)-r p L_{2} \sin \eta+\left(p^{2}+q^{2}\right) L_{2} \cos \eta\right) \\
& -M_{W}\left(\cos \eta \Phi_{3}^{\alpha}+\sin \eta\left(\mathbb{k}_{3}+2 q \varangle_{3}\right)-r p L_{3} \sin \eta-\left(p^{2}+q^{2}\right) L_{3} \cos \eta\right) \\
& -M_{W}\left(\cos \eta \otimes_{R}^{\alpha}+\sin \eta\left(\& \mathbb{k}_{4}+2 q \S_{R}\right)-r p L_{4} \sin \eta-\left(p^{2}+q^{2}\right) L_{4} \cos \eta\right) \\
& 0=-M_{W} g(\sin \theta \sin \psi \sin \phi+\cos \theta \cos \phi) \cos \eta\left(L_{1}+L_{2}-L_{3}-L_{4}\right)+M_{x} \\
& -M_{W} L_{1} \cos \eta\left(\alpha-\sin \eta\left(\alpha_{1}+r \otimes_{1}\right)+H \&+\cos \eta\left(\alpha_{1}+p \otimes_{1}\right)\right. \\
& \left.-p\left(w-\varangle_{1} \cos \eta-q\left(H-L_{1} \sin \eta\right)\right)+r\left(u-L_{1} \cos \eta q-\varangle_{1}^{\alpha} \sin \eta\right)\right)
\end{aligned}
$$

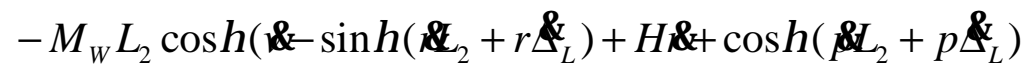

$$
\begin{aligned}
& \left.-p\left(w-\&_{L} \cos \eta-q\left(H-L_{2} \sin \eta\right)\right)+r\left(u-L_{2} \cos \eta q-\&_{L} \sin \eta\right)\right) \\
& +M_{W} L_{3} \cos \eta\left(\alpha-\sin \eta\left(\&_{3}+r \varangle_{3}\right)+H \&-\cos \eta\left(\alpha_{3}+p \otimes_{3}\right)\right. \\
& \left.-p\left(w+\otimes_{3} \cos \eta-q\left(H-L_{3} \sin \eta\right)\right)+r\left(u+L_{3} \cos \eta q-\$_{3} \sin \eta\right)\right)
\end{aligned}
$$

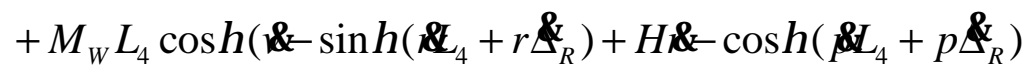

$$
\begin{aligned}
& \left.-p\left(w+\bigotimes_{R} \cos \eta-q\left(H-L_{4} \sin \eta\right)\right)+r\left(u+L_{4} \cos \eta q-\&_{R} \sin \eta\right)\right) \\
& -\mathbb{N}_{x}+\operatorname{qr}\left(I_{y}-I_{z}\right) \\
& -4\left(\cos ^{2} \eta \cdot I_{1}+q r \cos ^{2} \eta\left(I_{3}-I_{2}\right)+\sin ^{2} \eta \cdot \sin _{3}^{2} \eta\left(I_{1}-I_{2}\right)\right)
\end{aligned}
$$




$$
\begin{aligned}
& 0=M_{W} g \sin \theta \cos \psi \cos \eta\left(L_{1}+L_{2}-L_{3}-L_{4}\right) \\
& -M_{W} g(\sin \theta \sin \psi \cos \phi-\cos \theta \sin \phi)\left(\left(L_{1}+L_{2}+L_{3}+L_{4}\right) \sin \eta-4 H\right)+M_{y} \\
& +M_{W} L_{1} \cos \eta\left(\imath \&-\sin \eta \Phi_{1}^{\alpha}-\cos \eta\left(\phi \mathbb{k}_{1}+q \Delta_{1}\right)\right. \\
& \left.+q\left(w-\varangle_{1} \cos \eta-q\left(H-L_{1} \sin \eta\right)\right)-r\left(v+r\left(H-L_{1} \sin \eta\right)+p L_{1} \cos \eta\right)\right) \\
& +M_{W} L_{2} \cos \eta\left(L \&-\sin \eta \Phi_{L}-\cos \eta\left(\& \mathbb{L}_{2}+q \otimes_{L}\right)\right. \\
& \left.+q\left(w-\otimes_{L} \cos \eta-q\left(H-L_{2} \sin \eta\right)\right)-r\left(v+r\left(H-L_{2} \sin \eta\right)+p L_{2} \cos \eta\right)\right) \\
& -M_{W} L_{3} \cos \eta\left(\imath \&-\sin \eta \alpha_{3}+\cos \eta\left(\&_{3}+q \S_{3}\right)\right. \\
& \left.+q\left(w+\&_{3} \cos \eta-q\left(H-L_{3} \sin \eta\right)\right)-r\left(v+r\left(H-L_{3} \sin \eta\right)-p L_{3} \cos \eta\right)\right)
\end{aligned}
$$

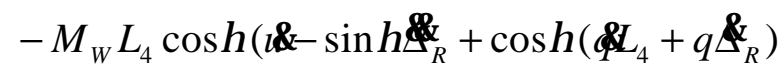

$$
\begin{aligned}
& \left.+q\left(w+\&_{R} \cos \eta-q\left(H-L_{4} \sin \eta\right)\right)-r\left(v+r\left(H-L_{4} \sin \eta\right)-p L_{4} \cos \eta\right)\right) \\
& -M_{W}\left(L_{1} \sin \eta-H\right)\left(1 \&-\cos \eta \Psi_{1}+\sin \eta\left(\phi \mathbb{k}_{1}+q \boldsymbol{\bigotimes}_{1}\right)-H \&\right. \\
& \left.+p\left(v+r\left(H-L_{1} \sin \eta\right)+p L_{1} \cos \eta\right)-q\left(u-q L_{1} \cos \eta-\&_{1} \sin \eta\right)\right) \\
& -M_{W}\left(L_{2} \sin \eta-H\right)\left(1 \ll-\cos \eta \Phi_{L}^{\alpha}+\sin \eta\left(\alpha_{2}+q \delta_{L}\right)-H \&\right. \\
& \left.+p\left(v+r\left(H-L_{2} \sin \eta\right)+p L_{2} \cos \eta\right)-q\left(u-q L_{2} \cos \eta-\&_{L} \sin \eta\right)\right)
\end{aligned}
$$

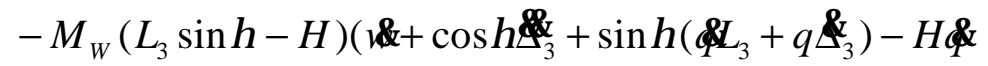

$$
\begin{aligned}
& \left.+p\left(v+r\left(H-L_{3} \sin \eta\right)-p L_{3} \cos \eta\right)-q\left(u+q L_{3} \cos \eta-\$_{3} \sin \eta\right)\right) \\
& -M_{W}\left(L_{4} \sin \eta-H\right)\left(1 \ll+\cos \eta \otimes_{R}+\sin \eta\left(\alpha_{4}+q \S_{R}\right)-H \&\right. \\
& \left.+p\left(v+r\left(H-L_{4} \sin \eta\right)-p L_{4} \cos \eta\right)-q\left(u+q L_{4} \cos \eta-\&_{R} \sin \eta\right)\right) \\
& -\phi \alpha_{y}+r p\left(I_{z}-I_{x}\right) \\
& -4\left(\alpha_{2}+\cos 2 \eta \cdot \operatorname{pr}\left(I_{1}-I_{3}\right)\right) \\
& 0=M_{W} g(\sin \theta \sin \psi \sin \phi+\cos \theta \cos \phi)\left(\left(\left(L_{1}+L_{2}+L_{3}+L_{4}\right) \sin \eta-4 H\right)\right)+M_{z} \\
& -M_{W}\left(H-L_{1} \sin \eta\right)\left(\&-\sin \eta\left(\mathcal{E}_{1}+r \otimes_{1}^{\alpha}\right)+H \&+\cos \eta\left(\mathbb{K}_{1}+p \otimes_{1}^{\alpha}\right)\right. \\
& \left.-p\left(w-\otimes_{1}^{\alpha} \cos \eta-q\left(H-L_{1} \sin \eta\right)\right)+r\left(u-L_{1} \cos \eta q-\&_{1}^{\alpha} \sin \eta\right)\right) \\
& -M_{W}\left(H-L_{2} \sin \eta\right)\left(\&-\sin \eta\left(\mathbb{k}_{2}+r \S_{L}\right)+H \&+\cos \eta\left(\alpha_{2}+p \S_{L}\right)\right. \\
& \left.-p\left(w-\varangle_{L} \cos \eta-q\left(H-L_{2} \sin \eta\right)\right)+r\left(u-L_{2} \cos \eta q-\otimes_{L} \sin \eta\right)\right)
\end{aligned}
$$

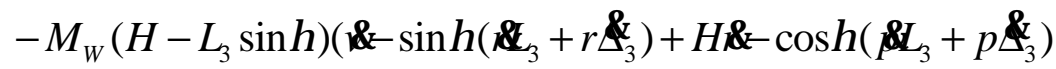

$$
\begin{aligned}
& \left.-p\left(w+\bigotimes_{3} \cos \eta-q\left(H-L_{3} \sin \eta\right)\right)+r\left(u+L_{3} \cos \eta q-\&_{3} \sin \eta\right)\right) \\
& -M_{W}\left(H-L_{4} \sin \eta\right)\left(\&-\sin \eta\left(\mathbb{E}_{4}+r \S_{R}\right)+H \&-\cos \eta\left(\mathbb{\alpha}_{4}+p \S_{R}\right)\right. \\
& \left.-p\left(w+\otimes_{R} \cos \eta-q\left(H-L_{4} \sin \eta\right)\right)+r\left(u+L_{4} \cos \eta q-\bigotimes_{R} \sin \eta\right)\right) \\
& -\delta \mathcal{E}_{z}+p q\left(I_{x}-I_{y}\right) \\
& -4\left(\sin ^{2} \eta \cdot \& I_{1}+p q \sin ^{2} \eta\left(I_{2}-I_{3}\right)+\cos ^{2} \eta \cdot \alpha_{3}+p q \cos ^{2} \eta\left(I_{2}-I_{1}\right)\right)
\end{aligned}
$$

In the practical flight, the aircraft's structure is symmetric about the central plane. So the aircraft's dynamic equations can be simplified to a set of simple dynamic equations with setting $\Delta_{1}=\Delta_{3} ; \Delta_{2}=\Delta_{4}$. 


$$
\begin{aligned}
& 0=-M_{T}(g \sin \theta \cos \psi+q \alpha w-r v)-D \cos \beta \cos \alpha+L \sin \alpha-Y \sin \beta \cos \alpha \\
& +2 M_{w}\left[2 H-\sin \eta\left(L_{1}+L_{2}\right)\right]\left(r^{2}+q^{2}\right)+2 M_{w} \sin \eta(\underbrace{}_{L}) \\
& 0=-M_{T}(g \sin \theta \sin \psi \sin \phi+g \cos \theta \cos \phi+\alpha w+r u)+D \cos \beta \sin \alpha+L \cos \alpha \\
& +Y \sin \beta \sin \alpha-2 M_{W}\left[2 H-\sin \eta\left(L_{1}+L_{2}\right)\right](\&-p q)+4 M_{W} r \sin \eta\left(\bigotimes_{1}+\bigotimes_{L}\right) \\
& 0=-M_{T}(g \sin \theta \sin \psi \cos \phi-g \cos \theta \sin \phi+1 \alpha+p v-q u)-D \sin \beta+Y \cos \beta \\
& -2 M_{W}\left[2 H-\sin \eta\left(L_{1}+L_{2}\right)\right]\left(r p-\phi-4 M_{W} q \sin \eta\left(\otimes_{1}+\bigotimes_{L}\right)\right. \\
& \delta=\left\{q r\left(I_{y}-I_{z}+4 \cos ^{2} \eta\left(I_{2}-I_{3}\right)+4 \sin ^{2} \eta\left(I_{2}-I_{1}\right)+2 M_{W} \cos ^{2} \eta\left(L_{1}^{2}+L_{2}^{2}\right)\right)\right. \\
& \left.-4 p M_{W} \cos ^{2} \eta\left(\otimes_{1} L_{1}+\varangle_{L} L_{2}\right)+M_{x}\right\} / I_{\text {xequ }} \\
& \oint \approx=\left\{r p\left[I_{z}-I_{x}-4 \cos 2 \eta\left(I_{1}-I_{3}\right)-2 M_{W}\left(L_{1}^{2}+L_{2}^{2}\right) \cos 2 \eta-4 M_{W} H \sin \eta\left(L_{1}+L_{2}\right)+4 M_{W} H^{2}\right]\right. \\
& -4 M_{W} q\left(L_{1} \otimes_{1}+L_{2} \S_{L}\right)+4 M_{W} q H \sin \eta\left(\otimes_{1}+\bigotimes_{L}\right) \\
& \left.-2 M_{W}(g \sin \theta \sin \psi \cos \phi-g \cos \theta \sin \phi+1 \kappa+p v-q u)\left(\left(L_{1}+L_{2}\right) \sin \eta-2 H\right)+M_{y}\right\} / I_{\text {yequ }} \\
& \delta\left\{\left\{p q \left(I_{x}-I_{y}-4 I_{2}+4 \sin ^{2} \eta I_{3}+4 \cos ^{2} \eta I_{1}-2 M_{W} \sin ^{2} \eta\left(L_{1}^{2}+L_{2}^{2}\right)+4 M_{W} H \sin \eta\left(L_{1}+L_{2}\right)\right.\right.\right. \\
& \left.-4 M_{W} H^{2}\right) \\
& -4 M_{W} r \sin ^{2} \eta\left(L_{1} \otimes_{1}+L_{2} \otimes_{L}\right)+4 M_{W} r H \sin \eta\left(\otimes_{1}+\bigotimes_{L}\right) \\
& +2 M_{W}(g \sin \theta \sin \psi \sin \phi+g \cos \theta \cos \phi+\alpha+u r-p w)\left(\left(L_{1}+L_{2}\right) \sin \eta-2 H\right) \\
& \left.+M_{z}\right\} / I_{z e q u} \\
& I_{\text {xequ }}=I_{x}+4 \cos ^{2} \eta I_{1}+4 \sin ^{2} \eta I_{3}+2 M_{W} \cos ^{2} \eta\left(L_{1}^{2}+L_{2}^{2}\right) \\
& I_{\text {yequ }}=I_{y}+4 I_{2}+2 M_{W}\left(L_{1}^{2}+L_{2}^{2}\right)+4 M_{W} H^{2}-4 M_{W} H \sin \eta\left(L_{1}+L_{2}\right) \\
& I_{\text {zequ }}=I_{z}+4 \sin ^{2} \eta I_{1}+4 \cos ^{2} \eta I_{3}+4 M_{W} H^{2}+2 M_{W} \sin ^{2} \eta\left(L_{1}^{2}+L_{2}^{2}\right)-4 M_{W} H \sin \eta\left(L_{1}+L_{2}\right)
\end{aligned}
$$

Where $I_{\text {xequ }}, I_{\text {yequ }}, I_{\text {zequ }}$ is the equivalent moment of inertia of the morphing aircraft. So far, we have obtained the six degrees of freedom dynamic equation of the morphing aircraft. The equations are different from the traditional single rigid body dynamic model, contains the additional force and the additional caused by the change of the position of the center of mass and the change of the moment of inertia.

\section{Controller Design}

In this chapter, auto disturbance rejection controller (ADRC) is adopted to design the controller, because:

The multi-rigid-body dynamic model of MGA is complex and uncertain, While the ADRC technology which adopts the idea of error feedback control doesn't need very accurate model [5].

The mass and aerodynamic characteristics of MGA vary significantly, while the ADRC compensates the total disturbance in real time through the extended state observer. When parameters of control object change or external disturbance exists, it can keep good control effect and has strong robustness.

The full dynamic equations of MGA can be simplified into the longitudinal equations by setting $\beta=0, \gamma=0, p=0, q=0$, then we transform the equations into a form suitable for ADRC design:

$$
\begin{aligned}
& \delta=f_{\alpha}+g_{1} \cdot \delta_{z} \\
& \alpha \delta=f_{r}+g_{2} \cdot r
\end{aligned} .
$$

Where, 


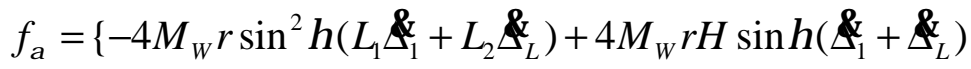

$$
\begin{aligned}
& \left.+2 M_{W}(g \cos \theta+1+u r)\left(\left(L_{1}+L_{2}\right) \sin \eta-2 H\right)+\left(C_{m z 0}+C_{m z, \alpha}+C_{m z, \omega_{z}}\right) Q S l\right\} / I_{z e q u} \\
& g_{1}=C_{m z, \delta_{z}} Q S l / I_{z e q u} \\
& f_{r}=-\frac{1}{M_{\text {total }} V}\left(L+M_{\text {total }} g \cos \vartheta\right) \\
& g_{2}=1 \\
& I_{z e q u}=I_{z}+4 \sin ^{2} \eta I_{1}+4 \cos ^{2} \eta I_{3}+4 M_{W} H^{2}+2 M_{W} \sin ^{2} \eta\left(L_{1}^{2}+L_{2}^{2}\right) \\
& -4 M_{W} H \sin \eta\left(L_{1}+L_{2}\right)
\end{aligned}
$$

The control variable is $\delta_{z}$, and the state variable is $\alpha, r$, where $\alpha$ is a slow variable and $r$ is a fast variable. For easy ADRC design, the control system is divided into fast contro loop (inner loop control loop) and slow control loop (outer control loop), as is shown in Figure 2. In the outer loop, the controlled variable is $\alpha$ and the control variable is $r$. The controlled variable is $r$ and the control variable is $\delta_{z}$ in the inner control loop.

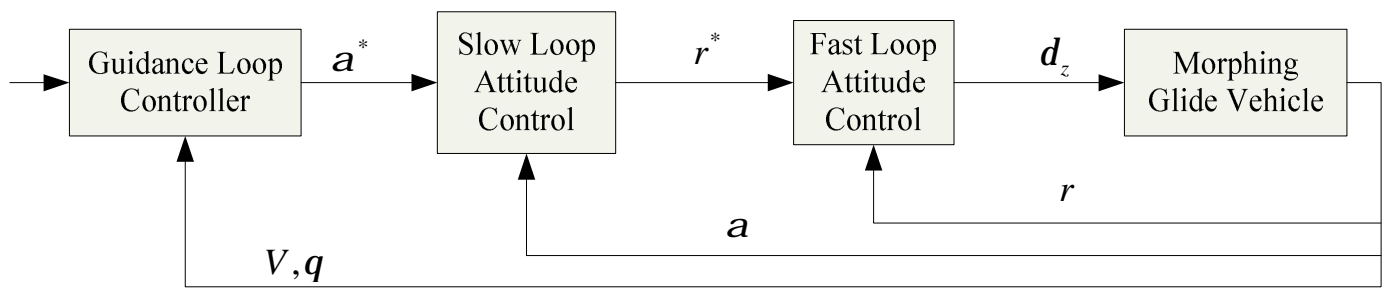

Figure. 3 Control loop

The extended state observer and nonlinear error feedback law for fast loop and slow loop are both designed as follows:

$$
\begin{aligned}
& \left\{\begin{array}{l}
e_{11}=z_{11}-\alpha \\
\delta_{11}=z_{12}-\beta_{01} e_{11}+U_{1} \\
\alpha_{12}=-\beta_{02} f a l\left(e_{11}, \alpha_{01}, \delta_{01}\right)
\end{array},\left\{\begin{array}{l}
e_{21}=z_{21}-r \\
\delta_{1}=z_{22}-\beta_{1} e_{21}+U_{2} \\
\alpha_{22}=-\beta_{2} f a l\left(e_{21}, \alpha_{1}, \delta_{1}\right) .
\end{array}\right.\right. \\
& \left\{\begin{array}{l}
e_{12}=\alpha^{*}-\alpha \\
U_{10}=\beta_{03} f a l\left(e_{12}, \alpha_{02}, \delta_{02}\right)
\end{array},\left\{\begin{array}{l}
e_{22}=r^{*}-r \\
U_{20}=\beta_{3} f a l\left(e_{22}, \alpha_{2}, \delta_{2}\right)
\end{array}\right.\right. \\
& \operatorname{fal}(e, \alpha, \delta)=\left\{\begin{array}{c}
|e|^{\alpha} \operatorname{sign}(e),|e|>\delta \\
e / \delta^{1-\alpha},|e| \leq \delta
\end{array} \delta q_{\alpha}=q_{\alpha}^{*}-q_{\alpha}(\alpha=1,2 \mathrm{~L} \mathrm{k)} .\right.
\end{aligned}
$$

Where $\beta_{01} 、 \beta_{02} 、 \alpha_{01} 、 \delta_{01} 、 \beta_{03} 、 \alpha_{02} 、 \delta_{02} 、 \beta_{1} 、 \beta_{2} 、 \alpha_{1} 、 \delta_{1} 、 \beta_{3} 、 \alpha_{2} 、 \delta_{2}$ are design parameter. Compared with PID and other control methods, ADRC needs to set more parameters, which affects each other and makes it difficult to adjust the parameters. In this paper, particle swarm optimization (PSO) algorithm is adopted to optimize the controller parameters $\beta_{1}, \beta_{2}, \alpha_{1}, \delta_{1}$, Objective function is integration of the error of the extended state observer. Controller parameters optimized are $\beta_{1}=1063.78, . \beta_{2}=1983.13, \alpha_{1}=0.2296, \delta_{1}=0.004296$. 


\section{Simulation Analysis}

The aircraft parameters and flight condition parameter are given as follows:

$$
\begin{aligned}
& M_{F}=1550 \mathrm{~kg}, M_{W}=30 \mathrm{~kg}, I_{z}=2612 \mathrm{~kg} \cdot \mathrm{m}^{2}, I_{1}=50 \mathrm{~kg} \cdot \mathrm{m}^{2}, I_{2}=20 \mathrm{~kg} \cdot \mathrm{m}^{2}, I_{3}=50 \mathrm{~kg} \cdot \mathrm{m}^{2}, \\
& S=0.4536 \mathrm{~m}^{2}, l=4.69 \mathrm{~m}, l_{1}=0.688, \mathrm{~L}=1.5968, M a=6, \mathrm{~h}=30 \mathrm{~km}
\end{aligned}
$$

The simulation parameters of ADRC controller are given as follows:

$\delta_{2}=0.1, \alpha_{2}=1.5, \beta_{3}=2000$,

$\delta_{02}=0.0001, \alpha_{02}=0.8, \beta_{03}=5$,

$\beta_{1}=1063.78, \beta_{2}=1983.13, \alpha_{1}=0.2296, \delta_{1}=0.004296$

$\beta_{01}=300, \beta_{02}=1000, \delta_{01}=0.05$

The expected angle of attack is $\alpha^{*}=3 \operatorname{sign}(\sin (t))+7$. We verify the stability of the control system when the morphing wings are retracted at a constant speed from the maximum wingspan to minimum wingspan. The transition time is 2.5 seconds. The total stretch length $\Delta$ of morphing wing is expressed as equation(15) and shown in Fig. 4.

$$
\Delta=\left\{\begin{array}{l}
1.376,0<t<2 \\
0.5504 *(t-2), 2<t<4.5 . \\
0, t>4.5
\end{array}\right.
$$

As shown in Fig 5, the ADRC can track the desired command signal almost without overshoot before, after and during the morphing progress.

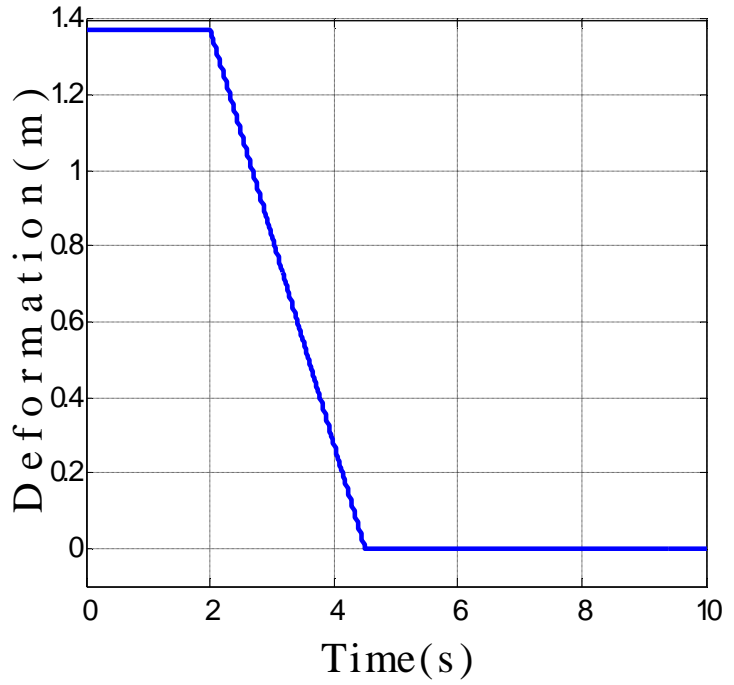

Fig. 4 Deformation curve

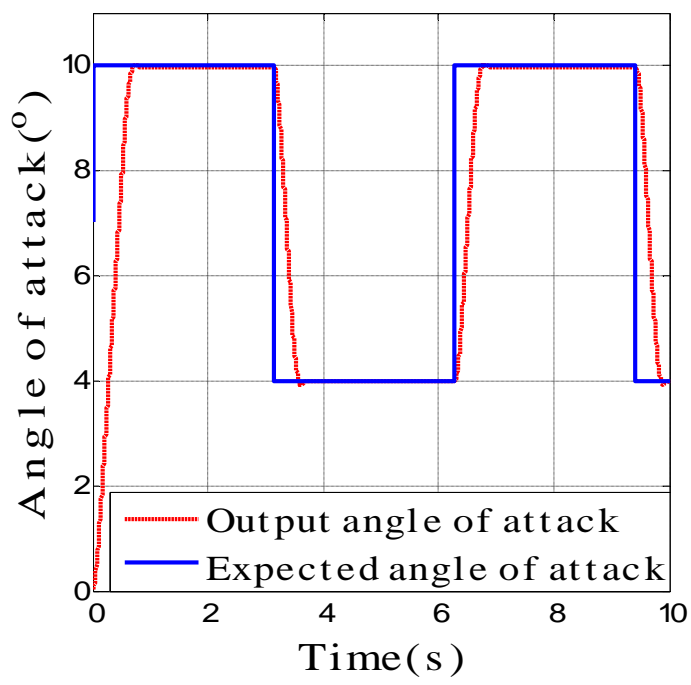

Fig. 5 Angle of attack curve

As shown in Fig 6, extended state observer can estimates disturbance signals and compensate the model accurately and ensure that controller has good anti-interference performance. 


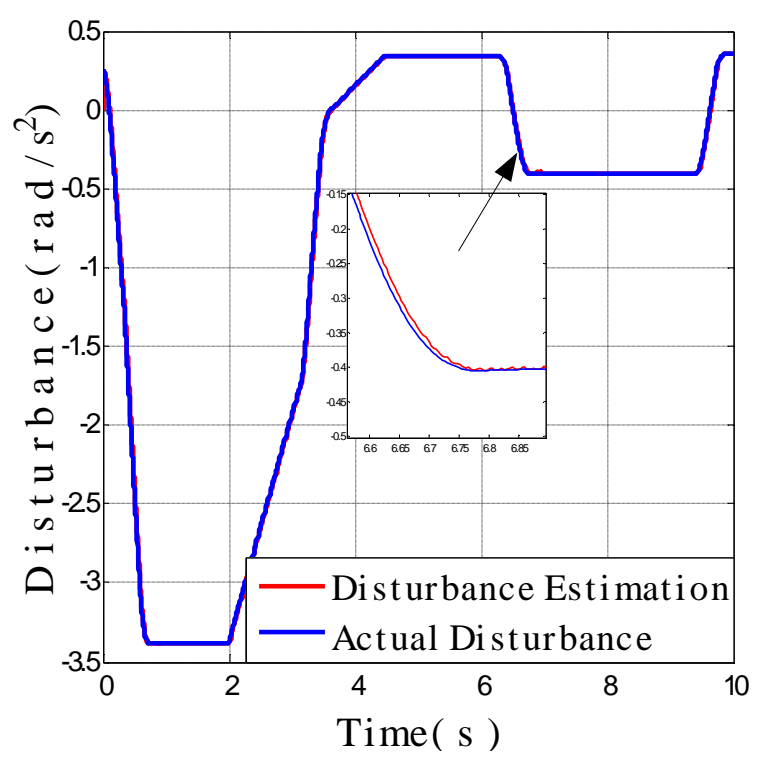

Fig. 6 Disturbance estimation curve

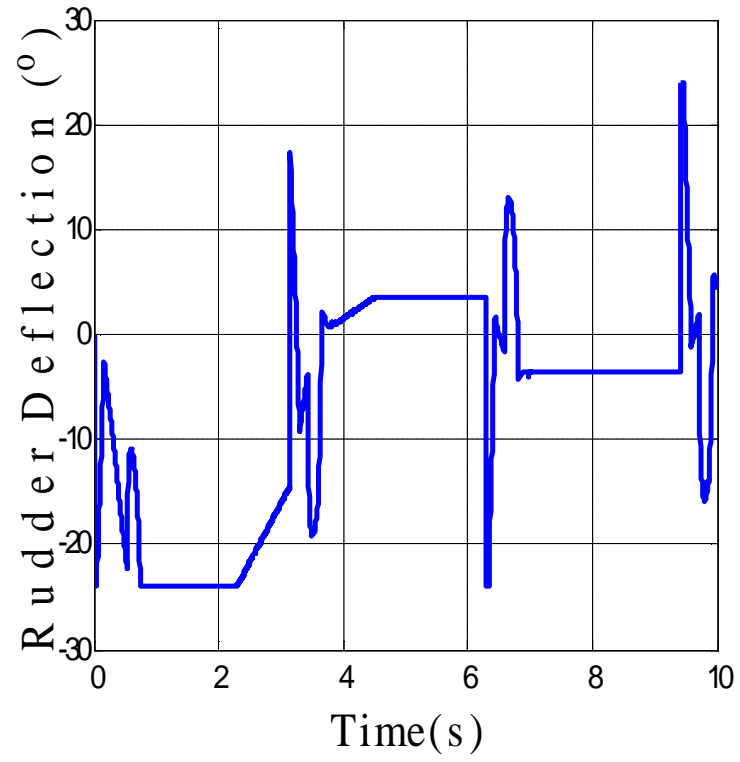

Fig. 7 Tiller angle curve

\section{Conclusion}

This article introduces the morphing requirement of near-space glide aircraft, and proposes a morphing aircraft which can change its wingspan. Based on Kane's Method, dynamic model of MGA is built. The model can be used as a basis for the morphing aircraft. The automatic disturbance rejection control technique is used to realize the stability control of MGA longitudinal model. PSO algorithm is adopted to optimize the control parameters. The simulation results show that the state observer and the nonlinear error feedback control law can track the input signal quickly and stably before, during and after morphing progress.

\section{References}

[1] Neha G, Akhilesh J, Jeffrey M. Intelligent control of a morphing aircraft[R]. AIAA-2007-1716, 2007.

[2] Mujahid A, Rick L. Control and simulation of a multi-role morphing micro air vehicle[R]. AIAA-2005-6481, 2005.

[3] Adam M W, Ephrahim G. Optimization of perching maneuvers through vehicle morphing[J]. Journal of Guidance, Control, and Dynamics, 2008, 31(4): 815-823.

[4] Chaohui Qi. Multibody system dynamics[M]. Science Press, 2008.

[5] Jingqing Han. Active Disturbance Rejection Control Technique[M]. National Defense Industry Press, 2008. 